\title{
Análise do número de cesarianas realizadas em uma maternidade após a aprovação da lei no Estado do Paraná
}

\author{
Analysis of the number of cesarean sections performed in a maternity hospital after the \\ approval of the law in the State of Paraná
}
Análisis del número de cesáreas realizadas en una maternidad después de la aprobación de la ley en el Estado de Paraná

Laura Edla Rönau Hadlich ${ }^{1 *}$, Natalia Albano Wendramin ${ }^{1}$, Paula Haus de Oliveira ${ }^{1}$, Rosangela Ramirez Taborda ${ }^{1}$, Somaia Reda ${ }^{1}$, Daiane Cristina Pazin ${ }^{1}$.

\section{RESUMO}

Objetivo: Avaliar a hipótese de ter ocorrido um aumento no número de cesarianas após a implementação da Lei n. 20.127, de 15/01/2020, assim como o impacto nos custos para o Sistema Único de Saúde (SUS). Métodos: Trata-se de um estudo observacional transversal, com coleta retrospectiva de dados em prontuários de 331 gestantes que realizaram cesariana eletiva, após a implementação da lei, e posteriormente, realizada uma comparação com o mesmo período de 2018 e 2019. As variáveis estudadas foram compiladas em arquivo, utilizando o programa Microsoft Excel® para posterior análise estatística através do teste de proporção, adotando-se significância $<0,05$. $O$ estudo foi aprovado por Comitê de Ética em Pesquisa. Resultados: Com relação ao número de cesarianas eletivas realizadas em 2020, concluiu-se que houve um aumento de $47,18 \%$ na taxa de cesarianas e redução de $18,6 \%$ dos partos vaginais, quando comparadas ao ano de 2018. Ainda, no tocante ao custo, observou-se uma elevação de $R \$ 32.305,71$ ao SUS. Conclusão: Observa-se, portanto, que há uma tendência na sociedade à realização de cesáreas, principalmente após a implementação da referida lei, bem como um maior custo ao serviço público.

Palavras-chave: Cesariana, Parto vaginal, Gestante, Sistema Único de Saúde, Legislação.

\section{ABSTRACT}

Objective: Evaluate the hypothesis that there was an increase in the number of cesarean sections after the implementation of Law n. 20.127 of 01/15/2020, as well as the impact on costs for the Sistema Único de Saúde (SUS). Methods: This is a cross-sectional observational study, with retrospective data collection from medical records of 331 pregnant women who underwent elective cesarean after the implementation of the Law. and, subsequently, a comparison to the same period in 2018 and 2019 was performed. The studied variables were compiled in a file, using the Microsoft Excel® program for further statistical analysis using the proportion test, adopting significance $<0.05$. The study was approved by the Research Ethics Committee. Results: Regarding the number of elective cesarean sections performed in 2020, it was concluded that there was an increase of $47.18 \%$ in the rate of cesarean sections and a reduction of $18.6 \%$ in vaginal deliveries, when compared to 2018. Regarding the cost, there was an increase of $R \$ 32,305.71$ to the SUS. Conclusion: It is observed, therefore, that there is a tendency in society to perform cesarean sections, especially after the implementation of the aforementioned law, as well as a higher cost to public service.

Keywords: Cesarean section, Vaginal delivery, Pregnant woman, Health Unic System, Legislation.

${ }^{1}$ Universidade Positivo (UP), Curitiba - PR. *E-mail: laurahadlich@yhaoo.com.br 


\section{RESUMEN}

Objetivo: Evaluar la hipótesis de que hubo un aumento en el número de cesáreas luego de la implementación de la Ley n. 20.127, del 15/01/2020, así como el impacto en costos para el Sistema Único de Salud (SUS). Métodos: Se trata de un estudio observacional transversal, con recolección de datos retrospectivos de las historias clínicas de 331 gestantes que fueron sometidas a cesárea electiva, luego de la implementación de la ley, y posteriormente se realizó una comparación con el mismo período de 2018 y 2019. Las variables estudiadas se recopilaron en un archivo, utilizando el programa Microsoft Excel® para posterior análisis estadístico mediante la prueba de proporciones, adoptando significancia $<0.05$. El estudio fue aprobado por el Comité de Ética en Investigación. Resultados: En cuanto al número de cesáreas electivas realizadas en 2020 , se concluyó que hubo un aumento del $47,18 \%$ en la tasa de cesáreas y una reducción del $18,6 \%$ en los partos vaginales, en comparación con el año 2018, hubo un aumento de $R \$ 32.305,71$ al SUS. Conclusión: Se observa, por tanto, que existe una tendencia en la sociedad a realizar cesáreas, especialmente luego de la implementación de la citada ley, así como mayor costo para el servicio público.

Palabras clave: Cesárea, Parto vaginal, Gestante, Sistema Único de Salud, Legislación.

\section{INTRODUÇÃO}

A saúde da mulher é um tema cada vez mais discutido na sociedade contemporânea, tendo sua origem de longa data (ZUGAIB M e FRANCISCO RPV, 2016). Tão importante e relevante é o tema que, verificou-se, em 2020, uma taxa de mortalidade materna que soma 44.603 mortes das mais variadas causas, o que deixa explícito a importância de ser abordado os cuidados com a saúde das mulheres e principalmente no período gestacional (BRASIL, 2021). Por ser um problema de saúde pública e de grande impacto na sociedade, a Organização das Nações Unidas (ONU), nos anos 2000, publicou os oito objetivos do milênio, no qual a "melhoria da saúde das gestantes" era uma das metas (BRASIL, 2020).

Corroborando com o exposto, nota-se a urgente necessidade na implementação de mais medidas para o cuidado com a saúde das mulheres e gestantes, podendo ser constatada com a alarmante taxa de morte materna por causas diretas (hipertensão, hemorragias, infecção puerperal e aborto), a qual, no ano de 2020, atingiu um número de 630 mulheres até o mês de agosto (BRASIL, 2021).

Um dos pontos importantes que tem contribuído com as elevadas taxas de morbimortalidade é o número exagerado de cesarianas que estão sendo realizadas, sem indicação médica, e que contribuem diretamente no aumento de complicações maternas e neonatais e, portanto, na mortalidade (BARROS FC, et al., 2018). Importante relembrar que a cesariana, ou parto cesariano, é um procedimento médico cirúrgico, por meio do qual o obstetra, médico especialista da área, promove uma incisão na região abdominal e remove o feto. Já o parto vaginal, também chamado de parto normal, é aquele no qual o feto nasce por via vaginal, na grande maioria das vezes, sem necessidade de intervenção médica ou da equipe (ZUGAIB M e FRANCISCO RPV, 2016).

Levando em consideração esta antiga discussão, sobre os aspectos positivos e negativos da cesariana, têm-se diversas entidades muito respeitadas, bem como a Federação Brasileira das Associações de Ginecologia e Obstetrícia (FEBRASGO), International Federation of Gynecology and Obstetrics (FIGO), Organização Mundial da Saúde (OMS), Sistema Único de Saúde (SUS), que concordam em importantes pontos, como a necessidade de utilizar-se tal método com foco nos casos em que há indicação médica, lançando mão, por exemplo, da classificação de Robson para indicar ou não a cesariana (BEZERRA LF, et al., 2019). Para isso, em 2015, a OMS declarou que pretende utilizar a Classificação de Robson, por ser facilmente aplicável, para avaliar, monitorar e comparar taxas de cesárea ao longo do tempo e, consequentemente, reduzir as taxas de cesariana desnecessárias (BRASIL, 2015).

As cesarianas realizadas com indicação médica são de grande benefício às pacientes, ao passo que as que são realizadas sem indicação não apresentam evidências de benefícios adicionais (BARROS FC, et al., 2018). Uma grande preocupação da OMS é garantir que todas as mulheres que realmente necessitem dessa 
intervenção consigam ter acesso, sendo proposto uma taxa ideal de até $15 \%$, embora saiba-se que o importante é o acesso das pacientes que realmente necessitam da intervenção, ao invés de se postular uma taxa ideal, muitas vezes, inatingível. Apesar disso, chama atenção à taxa nacional de 55\%, bem acima do preconizado, ocupando o $2^{\circ}$ lugar no ranking mundial e ficando atrás apenas da República Dominicana com $56 \%$ de taxa de cesariana (BETRAN AP, et al., 2016).

Segundo o Projeto Parto Adequado, no qual foram levantados 4 pontos para explicar o excesso de cesarianas no Brasil, nos últimos 30 anos, avaliou-se como relevante a conveniência para o obstetra, a prática obstétrica autônoma e o modelo de cuidado obstétrico que proporciona a medicalização do nascimento, ou seja, a submissão do binômio materno-fetal aos cuidados inteiramente hospitalares e intervencionistas (BOREM P, et al., 2020). Uma recente série de trabalhos publicados pela revista The Lancet alerta para o número excessivo de cesarianas e atribui essas taxas ao aumento do número de nascimentos em instituições de saúde e a maior frequência de cesarianas dentro das instituições (BOERMA T, et al., 2018). Adicionalmente, há a relação entre o nível de escolaridade e a escolha da via de parto que nos grupos com menor escolaridade a taxa de cesarianas chega a 19,4\%, enquanto em mulheres com nível educacional maior há um aumento para 54,4\% (HOXHA I, et al., 2017).

Mesmo com este cenário, no início de 2020, foi aprovada a Lei n. 20.127, de 15/01/2020, no Estado do Paraná, que alterou a Lei n. 19.701, de 20/11/2018 que dispõe sobre a violência obstétrica, sobre os direitos da gestante e da parturiente (PARANÁ, 2020). Consta no recente texto:

"Nas situações eletivas é direito da gestante optar pela realização da cesariana, desde que tenha recebido todas as informações pormenorizadas e que a cesariana a pedido da gestante, nas situações de risco habitual, somente poderá ser realizada a partir da 39a semana de gestação, devendo o registro estar presente no prontuário (PARANÁ, 2020).

Amparadas por essa lei, as gestantes têm aumentado substancialmente os pedidos para realização de cesariana, o que gera um fenômeno chamado de contraprodutividade, ou seja, uma ferramenta que passa a produzir efeitos paradoxais. A contraprodutividade entende que a cesariana, que deveria ser utilizada para partos difíceis e com indicações precisas, está sendo utilizada de forma irracional e causando malefícios às pacientes, como por exemplo, a iatrogenia (GOMES SC, et al., 2018).

Desta maneira, pretendeu-se avaliar a hipótese de ter ocorrido um aumento no número de cesarianas após a implementação da Lei n. 20.127 de 15/01/2020, assim como o impacto nos custos para o SUS. Adicionalmente, traçar relações com características gestacionais e maternas.

\section{MÉTODOS}

Trata-se de um estudo observacional transversal, com coleta retrospectiva de dados em prontuários de 331 gestantes que realizaram cesariana, com ou sem indicação médica, após a implementação da Lei $n$. 20.127, de 15/01/2020, em uma maternidade no Estado do Paraná, obtidas por meio de uma amostra de conveniência. Para fins de comparação de dados, realizou-se a coleta dos nascimentos (valor numérico) nos anos de 2018, 2019 e 2020. Aprovado pelo Comitê de Ética em Pesquisa (CEP) da Universidade Positivo, parecer número 4.070.667; Certificado de Apresentação de Apreciação Ética (CAAE) 30950520.7.0000.0093. Além da aprovação pelo CEP do Hospital do Trabalhador, parecer número 4.112.514; CAAE 30950520.7.3002.5225.

Os critérios de inclusão foram todas as gestantes que realizaram cesariana, com ou sem indicação médica, de 15 de janeiro de 2020 a 15 de setembro de 2020. Os critérios de exclusão deste estudo foram pacientes que realizaram parto vaginal e prontuários com dados incompletos.

Foram consideradas cesarianas com indicação médica: pacientes com idade gestacional < 39 semanas; pacientes submetidas ao procedimento devido a urgência ou emergência; cesariana iterativa ( $\geq 2$ cesarianas anteriores); motivos fetais. Por outro lado, foram consideradas cesarianas sem indicação médica: todas aquelas realizadas a pedido da paciente, de acordo com a Lei n. 20.127, de 15/01/2020. 
As variáveis estudadas foram coletadas dos prontuários e compiladas em arquivo, utilizando o programa Microsoft Exce ${ }^{\circledR}$ para posterior análise estatística através do teste de proporção, essa considerada $1 / 3$ a cada ano, e adotando-se significância $<0,05$.

Para descrição do perfil das gestantes e variáveis da gestação foram investigados o número do prontuário, idade da gestante, paridade, número de gestação, partos vaginais, cesarianas, abortos e gestações ectópicas, se houve intercorrências no último parto, gestação atual múltipla ou não, idade gestacional ecográfica, drogadição e vícios, comorbidades clínicas, presença de complicação na cesariana e a justificativa para a realização da cesariana (urgência ou emergência, decisão médica, iteratividade, motivo fetal, a pedido da gestante).

\section{RESULTADOS}

Com relação aos dados epidemiológicos das 331 gestantes, observou-se uma idade média de 27,37 anos. Destas, 88 eram primigestas e 243 eram multigestas. Dentre todas as gestantes, a média do número de gestações foi de 2,32. Notou-se que 75 gestantes possuíam histórico de aborto e 4 de gestação ectópica, sendo que 2 destas eram do grupo que optou pela cesárea a pedido. Ao observar os dois grupos de forma distinta, grupo de cesarianas a pedido e por indicação médica, constata-se que a média da idade das gestantes do primeiro grupo foi de 26 anos, já a do segundo grupo foi de 27,71 anos.

No ano de 2018 houve 2.461 nascimentos, dentre os quais 1.734 foram partos via vaginal e 727 por parto cesariano. Em 2019, ocorreram 2.514 partos, sendo 1.667 vaginais e 847 cesarianas. Por fim, em 2020, observou-se o número de 2.480 nascidos, dos quais 1.410 foram via vaginal e 1.070 via cesariana. Foi observado, por meio da comparação de dados destes 3 anos, um aumento de 47,18\% de cesarianas do ano 2018 para o ano de 2020 , apresentando $p<0,001$. Ao passo que os partos via vaginal diminuíram $18,6 \%$ entre esses mesmos anos.

Dos partos cesarianos, realizados em 2020, foram analisados 331 prontuários de gestantes que realizaram cesariana eletiva em uma maternidade do Estado do Paraná. Destes, $115(34,7 \%)$ foram partos cesarianos a pedido materno e $216(65,2 \%)$ foram cesarianas com indicação médica, estão incluídas as cesarianas realizadas por motivos fetais ou motivos maternos.

$\mathrm{Na}$ Tabela 1 elencou-se os dados sobre a idade gestacional das pacientes submetidas à cesariana por indicação médica, e, também, a relação com a idade materna.

Tabela 1 - Perfil da IG* e da idade materna, com relação às cesarianas com indicação médica e a pedido materno.

\begin{tabular}{ccccc}
\hline \multicolumn{2}{c}{ VARIÁVEIS ESTUDADAS } & $\begin{array}{c}\text { COM INDICAÇÃO } \\
\text { MÉDICA }\end{array}$ & A PEDIDO MATERNO & TOTAL \\
\hline \multirow{2}{*}{ Idade Gestacional } & $\geq 39$ SEMANAS & $132(64,8 \%)$ & $115(100 \%)$ & 247 \\
& $<39$ SEMANAS & $76(35,1 \%)$ & 0 & 76 \\
\hline \multirow{2}{*}{ Idade materna } & $<35$ ANOS & $179(82,8 \%)$ & $110(95,6 \%)$ & 289 \\
& $\geq 35$ ANOS & $37(17,1 \%)$ & $5(4,3 \%)$ & 42 \\
\hline \multicolumn{2}{c}{ TOTAL } & $216(100 \%)$ & $115(100 \%)$ & 331 \\
\hline
\end{tabular}

Legenda: *IG: Idade Gestacional.

Fonte: Hadlich LER, et al., 2021.

Em relação à paridade, das que realizaram cesariana com indicação médica, 48 (22,2\%) eram primigestas, enquanto $40(34,7 \%)$ das gestantes que efetuaram o procedimento a pedido eram deste mesmo grupo. Com relação às comorbidades, os resultados estão apresentados na Tabela 2. 
Tabela 2 - Dados epidemiológicos de comorbidades.

\begin{tabular}{ccc}
\hline COMORBIDADES & $\begin{array}{c}\text { CESARIANA COM } \\
\text { INDICAÇÃO MÉDICA }\end{array}$ & $\begin{array}{c}\text { CESARIANA SEM } \\
\text { INDICAÇÃO MÉDICA }\end{array}$ \\
\hline HAS $^{*}$ & $7(3,2 \%)$ & $0(0 \%)$ \\
DM $^{*}$ & $2(0,9 \%)$ & $0(0 \%)$ \\
DMG $^{*}$ & $22(10,1 \%)$ & $0(0 \%)$ \\
Asma & $9(4,1 \%)$ & $2(1,7 \%)$ \\
Hipotireoidismo & $29(13,4 \%)$ & $14(12,1 \%)$ \\
Anemia & $27(12,5 \%)$ & $3(2,6 \%)$ \\
Pré-eclâmpsia sem sinais de gravidade & $20(9,25 \%)$ & $0(0 \%)$ \\
Pré-eclâmpsia com sinais de gravidade & $11(5 \%)$ & $0(0 \%)$ \\
ITU* $^{*}$ & $18(8,3 \%)$ & $6(5,2 \%)$ \\
IST $^{*}$ & $10(4,6 \%)$ & $2(1,7 \%)$ \\
\hline
\end{tabular}

Legenda: * Hipertensão arterial sistêmica (HAS), Diabetes Mellitus (DM), Diabetes Mellitus Gestacional (DMG), Infecção do Trato Urinário (ITU) e Infecção Sexualmente Transmissível (IST).

Fonte: Hadlich LER, et al., 2021.

\section{DISCUSSÃO}

A proposta deste artigo foi identificar se houve aumento significativo do número de cesarianas após a aprovação da Lei Paranaense n. 20.127, de 15 de janeiro de 2020. Desta forma, observou-se o aumento de $47,18 \%$ no número de cesarianas de 2018 a 2020 e de $26,32 \%$ ao comparar 2019 e 2020 . Por meio destes resultados, evidencia-se que há uma tendência no aumento de cesarianas, sobretudo, após a aprovação da referida lei. Para as 115 gestantes que optaram por cesariana, respaldadas pela norma legal, notou-se relativamente ao esperado, menor quantidade de complicações, que se pode atribuir, no âmbito teórico, à melhora da assistência à saúde da mulher desde o pré-natal, até o puerpério, ou seja, a lei contribuiu de modo significativo para o aumento de cesarianas (CÂMARA R, et al., 2016).

Em 2020, o Brasil ocupava o segundo lugar na classificação mundial em números de cesáreas, acima dos $50 \%$, quando comparado a países da Europa e Ásia, por exemplo, onde $25 \%$ dos bebês nascem por parto cesariano (CÂMARA R, et al., 2016; ALMEIDA L, 2020). Por sua vez, o Estado de São Paulo já havia discutido essa possibilidade de a gestante escolher a via do parto a partir das 39 semanas, sendo esta a Lei Paulista n. 17.137/2019 (ASSOCIAÇÃO DE OBSTETRÍCIA E GINECOLOGIA DO ESTADO DE SÃO PAULO (SOGESP), 2020).

Porém, em julho de 2020, esta lei foi considerada inconstitucional, por contrariar as diretrizes de saúde da mulher já existentes, e, além disso, aumentar os riscos para os recém-nascidos e para as mães. Até o presente momento, no Estado do Paraná a Lei n. 20.127, de 15/01/2020 ainda está em vigor, mesmo tendo existido no Estado vizinho o debate que culminou a derrubada daquela norma (SOGESP, 2020).

Com relação às cesarianas a pedido, em cumprimento à Lei n. 20.127, de 15/01/2020, sabe-se que é direito da gestante realizar cesariana a pedido se: (1) risco habitual; (2) $\geq 39$ semanas (3) com registro em prontuários (PARANÁ, 2020). Baseado nisso, observou-se que as 115 gestantes que realizaram cesariana a pedido cumpriram os requisitos da lei. Assim, de acordo com o Código de Ética Médica (CEM) é direito do médico: "indicar procedimento adequado ao paciente, observadas as práticas cientificamente reconhecidas e respeitando a legislação vigente". Logo, é fundamental que o profissional de saúde esteja alinhado com seus direitos e deveres, de acordo com a legislação vigente, para, assim, poder ofertar ao seu paciente o melhor tratamento e em conformidade com a Lei (CONSELHO FEDERAL DE MEDICINA, 2019).

De acordo com a tabela padronizada pelo SUS, o custo padrão, ou seja, aquele que leva em consideração partos sem complicações, é de $R \$ 434,40$ para o parto vaginal e $R \$ 545,73$ para o parto cesariano (DATASUS, 2021). Entretanto, estudos mostraram que o custo real do hospital com esses procedimentos é superior, tal como em um hospital federal, em 2009, de Minas Gerais, no qual observou-se o custo de $R \$ 954,58$ para parto normal e R\$1.244,99 para parto cesariano (ENTRINGER AP, et al., 2019). 
Fica evidente, portanto, a dificuldade de executar o gerenciamento hospitalar, visto que os custos reais do procedimento superam os valores repassados pelo SUS. O SUS, por ser universal e gratuito, oferece muitos serviços de saúde para mais de 190 milhões de pessoas em todo o Brasil e, assim, é compreensível que haja essa regulação da verba. Por esse motivo, é de suma importância abordar a aplicação de políticas públicas, como a Lei n. 20.127, de 15/01/2020, que também considerem a viabilidade dos recursos que serão empregados para seu desenvolvimento (PIRES MNB, et al., 2018).

Paradoxalmente ao aumento do número absoluto de cesarianas, percebeu-se que o custo atribuído a este procedimento não acarretou, no período avaliado, um gasto significativo. Visto que no gerenciamento hospitalar os valores são na casa de milhões, o custo excedente calculado foi de $\mathrm{R} \$ 32.305,71$, valor irrisório se comparado ao valor total. No entanto, é preciso lembrar que o período avaliado foi de 9 meses, logo após a implementação da lei e em apenas uma maternidade no Estado do Paraná. Transportando esses valores para nível estadual, é possível que haja um aumento mais significativo para as contas do Estado e, consequentemente, do país. Este aumento pôde ser percebido em um estudo realizado pela OMS, com 137 países tendo sido constatado que na metade dos países estudados houve um gasto excedente de 6,2 milhões com cesarianas desnecessárias, valor correspondente a cinco vezes mais do que o valor gasto com as cesarianas indicadas (ENTRINGER AP, et al., 2019).

Para possibilitar uma análise ainda mais completa sobre o tema, faz-se necessário, portanto, revisar este conceito futuramente, avaliando um período maior de cesarianas a pedido pautadas nesta legislação, bem como expandir a avaliação para nível estadual. Além disso, é necessário levar em consideração que um parto cesariano envolve riscos de complicações intra e pós-operatórias, como hemorragia puerperal, infecção pósparto, internações em UTI e morte materna, elevando, dessa forma, ainda mais os custos relacionados a esse procedimento (MASCARELLO KC, et al., 2017). Outro fator que eleva potencialmente os gastos do sistema de saúde relacionados ao parto cirúrgico é dado pelo "efeito cascata" das cesarianas, no qual o aumento das taxas de cesáreas eleva o número de mulheres com cesariana anterior, que, por consequência, são mais propensas a realizar cesarianas de repetição (FERREIRA RNF e NASCIMENTO GQF, 2021).

Neste contexto, e visando a prestação de serviço de saúde para toda a população brasileira, o Estado recorre à chamada tese da Reserva do possível. De origem alemã, o princípio da teoria era a discussão sobre o limite do número de vagas nas universidades públicas alemãs. O Tribunal alemão entendeu que a prestação de serviços, por parte do Estado, deve seguir um limite razoável, de acordo com os recursos disponíveis para as demandas solicitadas pelos indivíduos (SILVA IA e BENACCHIO M, 2018).

Já no Brasil, transformou-se a ideia em uma "teoria da reserva do financeiramente possível". Dessa forma, tal tese só pode ser aplicada para a execução dos direitos sociais em caso de evidência de falta de recursos financeiros para tal (SILVA IA e BENACCHIO M, 2018; MÂNICA FB, 2017). Esse entendimento é o próprio reflexo da teoria, pois é irrefutável que as necessidades são imensas e os recursos para supri-las são exíguos (MÂNICA FB, 2017).

A aplicabilidade desta tese, neste contexto, reside na destinação de recursos públicos direcionado às demandas mais urgentes, considerando primordialmente àquelas que coloquem em risco a vida e a saúde dos pacientes, em detrimento de outras que são meramente eletivas, logo, decorrem de anseios e deliberação de cada indivíduo (SILVA IA e BENACCHIO M, 2018; MÂNICA FB, 2017).

Levando em consideração a interpretação da Teoria da Reserva do Possível, para a Lei n. 20.127, de 15/01/2020, o acesso indiscriminado à cesariana torna-se uma demanda fora dos critérios de indicação médica e custosa ao SUS (MÂNICA FB, 2017). Tendo em vista que para todas as pacientes que possuem indicação para realizar cesariana o sistema fornece este serviço, para as demais, que não necessitam, é ofertado o parto vaginal - menos custoso e com menos complicações. Assim, quando o Estado está diante de uma solicitação de demanda, cuja prestação do serviço pode alterar a história natural da doença para aquele paciente, é dever daquele fornecer o serviço (BRASIL, 2020).

No entanto, para o caso específico das cesarianas a pedido, esta demanda poderia ser considerada como um serviço que não mudará a história natural da gestação, ou seja, o parto ocorrerá de forma vaginal, na 
grande maioria das vezes, sem necessidade de intervenções cirúrgicas. É por esta ótica que o Estado utiliza a argumentação apontada pela Teoria da Reserva do Possível, objetivando mirar os recursos financeiros nas demandas mais urgentes ou que podem mudar drasticamente a repercussão da doença (BRASIL, 2020).

Um importante contraponto sobre o assunto é a análise do tema sob a perspectiva da autonomia da mulher, dada a importância de garantir à paciente o acesso à informação, pormenorizada, sobre a via de parto, durante o pré-natal, a fim de que compreenda os riscos e benefícios tanto do parto vaginal, quanto cesário (MASCARELLO KC, et al., 2017). Porém, dentro do contexto de escolha da via de parto na rede pública, não podemos esquecer que o Estado tem como dever fornecer atendimento à saúde para toda a população, de forma que não falte recurso para a perpetuação deste serviço gratuito e universal para todos (BRASIL, 2020).

A discussão, proposta neste tópico, é sobre a importância e a necessidade do acesso ao conhecimento sobre gestação, parto e puerpério, para todas as gestantes e, a forma como esse conhecimento pode fazer diferença na maneira como o parto vaginal é, culturalmente, entendido pelas mulheres. Assim, é imprescindível relembrar que a luta para reduzir a mortalidade materna foi, e continua a ser, extenuante e fazse necessário estar atento aos pequenos indícios de que possa haver aumento. Preservar a saúde da mulher, evitar complicações a ela e ao recém-nato, bem como, preservar a continuidade do SUS, são tarefas fundamentais para a prevenção e promoção da saúde dos brasileiros. Por esse motivo, os princípios da universalidade, integralidade e longitudinalidade devem sempre estar à frente das políticas públicas, para que seja possível fornecer acesso de qualidade para todos os brasileiros que utilizam, exclusivamente, o SUS.

\section{CONCLUSÃO}

Nota-se a existência de uma tendência à realização de cesarianas no Brasil, constatadas pelo aumento significativo dos dados nesse sentido. Aliado a isso, a publicação da Lei Paranaense n. 20.127, de 15/01/2020, surge dando respaldo à prática desta abordagem cirúrgica, a qual, muitas vezes, não é necessária. Se pensarmos nesse aumento a longo prazo, políticas públicas de incentivo ao parto vaginal terão sido em vão. Sob o aspecto do custo excedente ao sistema de saúde, no período analisado da pesquisa (9 meses), não foi possível avaliar aumento orçamentário significativo especificamente ao hospital público de Curitiba envolvido. Entretanto, se o foco passar a ser do ponto de vista mundial e utilizar os dados obtidos através de estudo realizado pela OMS com 137 países, infere-se o aumento dos gastos alcançando a casa dos milhões. Portanto, pode-se verificar que, a longo prazo, a implementação de leis como a do Estado do Paraná (Lei n. 20.127), podem contribuir para que haja um impacto financeiro expressivo para o sistema de saúde pública.

\section{REFERÊNCIAS}

1. BARROS FC, et al. Caesarean sections and the prevalence of preterm and early-term births in Brazil: secondary analyses of national birth registration. BMJ Open, 2018; 8: 1-9.

2. BETRÁN AP, et al. The Increasing Trend in Caesarean Section Rates: Global, Regional and National Estimates: 19902014. PLoS One, 2016; 11(2): 1-12.

3. BEZERRA LF, et al. Classificação de Robson nas maternidades brasileiras: estatísticas prós e contras. Revista Remecs, 2019; 4(7): 38-46.

4. BOERMA T, et al. Global epidemiology of use of and disparities in caesarean sections. The Lancet, 2018; 392: 13411348.

5. BOREM P, et al. A Quality Improvement Initiative to Increase the Frequency of Vaginal Delivery in Brazilian Hospitals. Obstet Gynecol, 2020; 135(2): 415-425.

6. BRASIL. Declaração da OMS sobre taxa de cesáreas. 2015. Disponível em: https://www.unasus.gov.br/noticia/declaracao-da-oms-sobre-taxas-de-cesareas. Acessado em: 11 nov. 2020.

7. BRASIL. Objetivos de Desenvolvimento do Milênio. 2020. Disponível em: https://nacoesunidas.org/tema/odm/. Acessado em: 19 mar. 2020.

8. BRASIL. Sistema Único de Saúde (SUS): estrutura, princípios e como funciona. 2020. Disponível em: https://www.gov.br/saude/pt-br/assuntos/saude-de-a-a-z/s/sistema-unico-de-saude-sus-estrutura-principios-e-comofunciona. Acessado em: 26 de julho de 2021. 
9. BRASIL. Painel de Monitoramento da Mortalidade Materna. 2021. Disponível em: http://svs.aids.gov.br/dantps/centrais-de-conteudos/paineis-de-monitoramento/mortalidade/materna/. Acessado em: 11 nov. 2020.

10. CÂMARA R, et al. Cesariana a pedido materno. Rev. Col. Bras. Cir., 2016; 43(4): 301-310.

11. CONSELHO FEDERAL DE MEDICINA. Código de Ética Médica (CEM). Brasil, 2019. Disponível em: https://portal.cfm.org.br/images/PDF/cem2019.pdf. Acessado em: 25 de Abril de 2021.

12. DATASUS. SIGTAP: Sistema de Gerenciamento da Tabela de Procedimentos, Medicamentos e OPM do SUS. 2021. Disponível em: http://sigtap.datasus.gov.br/tabela-unificada/app/sec/inicio.jsp. Acessado em: 05 jul. 2021.

13. ENTRINGER AP, et al. Análise de custos da atenção hospitalar ao parto vaginal e à cesariana eletiva para gestantes de risco habitual no Sistema Único de Saúde. Ciência \& Saúde Coletiva, 2019; 24(4): 1527-1536.

14. FERREIRA RNF, NASCIMENTO GQF. Análise da taxa de cesarianas: estudo comparativo entre duas maternidades públicas no estado do Rio de Janeiro. Femina, 2021; 49(7): 414-420.

15. GOMES SC, et al. Rebirth of childbirth: reflections on medicalization of the Brazilian obstetric care. Rev Bras Enferm, 2018; 71(5): 2594-8.

16. HOXHA I, et al. Caesarean sections and for-profit status of hospitals: systematic review and meta-analysis. BMJ Open, $2017 ; 7(2): 1-9$.

17. MÂNICA FB. Teoria da reserva do possível: Direitos Fundamentais a prestações e a intervenção do Poder Judiciário na implementação de políticas públicas. Cadernos da Escola de Direito, 2017; 1(8): 89-104.

18. MASCARELLO KC, et al. Maternal complications and cesarean section without indication: systematic review and metaanalysis. Revista de Saúde Pública, 2017; 51: 105.

19. PARANÁ. Lei no 20.127, de 15 de janeiro de 2020. Altera a Lei oㅜ 19.701, de 20 de novembro de 2018, que dispõe sobre a violência obstétrica, sobre direitos da gestante e da parturiente e revoga a Lei ํㅜ 19.207, de 1 de novembro de 2017, que trata da implantação de medidas de informação e proteção à gestante e à parturiente contra a violência obstétrica. Diário Oficial do Estado do Paraná. Curitiba, PR, 15 jan. 2020. Disponível em: https://www.legisweb.com.br/legislacao/?id=388956. Acessado em: 19 mar. 2020.

20. PIRES MBN, et al. Relação entre a remuneração do Sistema Único de Saúde, os custos dos procedimentos hospitalares e o resultado: estudo nas Santas Casas de Misericórdia do Estado de São Paulo. Revista de Administração Hospitalar e Inovação em Saúde, 2018; 14(3): 16-33.

21. SILVA IA, BENACCHIO M. A conceituação da teoria da reserva do possível e do mínimo existencial: reflexões para a concretização da desjudicialização. Revista Direito Mackenzie, 2018; 12(2): 1-14.

22. SOGESP. LEI № 17.137/2019 é declarada inconstitucional pelo Tribunal de Justiça de São Paulo - TJSP. 2020. Disponível em: https://www.sogesp.com.br/noticias/lei-no-171372019-e-declarada-inconstitucional-pelo-tribunal-dejustica-de-sao-paulo-tjsp/. Acessado em: 26 jun. 2021.

23. ZUGAIB M, FRANCISCO RPV. Zugaib obstetrícia. 3. ed. Barueri: Manole, 2016; 02p. 